\title{
Grain boundary characterization from particle coordinates
}

\author{
Nicholas H. P. Orr $\odot,{ }^{1}$ Taiki Yanagishima $\odot,{ }^{1,2}$ Eric Maire $\odot,{ }^{3}$ and Roel P. A. Dullens $\oplus^{1,4, *}$ \\ ${ }^{1}$ Department of Chemistry, Physical and Theoretical Chemistry Laboratory, University of Oxford, South Parks Road, \\ Oxford OX1 3QZ, United Kingdom \\ ${ }^{2}$ Department of Physics, Graduate School of Science, Kyoto University, Kitashirakawa Oiwake-cho, Sakyo-ku, Kyoto, 606-8502, Japan \\ ${ }^{3}$ Université de Lyon, INSA-Lyon, MATEIS, CNRS UMR5510, F-69621 Villeurbanne, France \\ ${ }^{4}$ Institute for Molecules and Materials, Radboud University, Heyendaalseweg 135, 6525 AJ Nijmegen, The Netherlands
}

(Received 30 April 2021; accepted 26 October 2021; published 17 December 2021)

\begin{abstract}
We present a method to locate and characterize grain boundaries in polycrystalline materials from the real-space coordinates of their constituent particles. The method is robust against imperfections such as thermal noise and vacancies. We apply the algorithm to experimentally found real-space coordinates to explicitly measure local misorientations and structure at grain boundaries. We consider particle coordinates obtained from an epitaxially templated colloidal $\Sigma 17$ bicrystal, finding that, even though the bicrystal is predominantly $\Sigma 17$ and face-centered cubic, small volumes of hexagonally closed-packed structure generate a mosaic of grain boundaries, increasing the complexity of the templated grain boundary. We also consider a homogeneously nucleated colloidal polycrystal and apply our method with no prior knowledge of grain boundary structure. Accordingly, we reveal detailed misorientation distributions and grain boundary structures. The method may be applied to any set of coordinates of atoms or particles in a polycrystalline system.
\end{abstract}

DOI: 10.1103/PhysRevMaterials.5.123605

\section{INTRODUCTION}

Most crystalline materials in nature are polycrystalline, consisting of differently oriented crystallites packed together to create a network of grain boundaries at their interfaces. The structure of the grain boundary network has a profound impact on the macroscopic material properties and underlie much of our understanding of ductility, brittleness, electrical conductivity, deformation and fracture mechanisms [1], melting kinetics [2-4], and transport properties [5] in a wide range of materials [6,7]. Much effort has been made in recent decades to tune the grain boundary structure within materials by "grain boundary engineering," which can involve sintering, rolling, and annealing of metals, alloys, and ceramics. In some cases, materials with small grains and many grain boundaries are desirable as they cause a dramatic increase in strength following the Hall-Petch law [8,9]. In other cases, large single crystals are required, with examples including structure solution in single crystal crystallography and the manufacture of photonic band-gap crystals [10-12].

The properties of the grain boundaries themselves are governed by their degrees of freedom. In three dimensions these consist of three for position, three for the orientation difference between the different crystallites, termed misori-

\footnotetext{
*Corresponding author: roel.dullens@ @ru.nl

Published by the American Physical Society under the terms of the Creative Commons Attribution 4.0 International license. Further distribution of this work must maintain attribution to the author(s) and the published article's title, journal citation, and DOI.
}

entation, and two for the plane of the boundary relative to the crystal lattice, known as inclination [13]. The distribution and type of grain boundary has been shown to affect the migration kinetics of boundaries under stress [14] and during grain growth $[15,16]$. Additionally, grain boundary mobility, surface tension, and roughening transitions are affected by the interplay between misorientation and inclination [17].

A wealth of research on materials yields structural information in reciprocal space using scattering techniques, such as orientation imaging microscopy $[18,19]$, diffraction contrast microscopy [20-23], electron backscatter diffraction [24,25], and three-dimensional (3D) x-ray diffraction $[26,27]$. However, one is either required to destroy the sample by serial sectioning, preventing dynamic measurements, or to prioritize between spatial, angular, or time resolution [28]. The spatial resolution of these techniques limits the size of the grains to roughly $1 \mu \mathrm{m}[23,28]$, orders of magnitudes larger than the size of the atoms themselves, making it impossible to comment on how the behavior of specific boundaries are tied to atomistic dynamics or arrangements. Furthermore, achieving measurements of grain boundaries in the bulk is difficult as one must have a sample thick enough to render surface effects negligible, but not so thick that scattering peaks from multiple grains overlap [28]. Thus, there are significant advantages to studying the real-space coordinates of atoms, molecules, or mesoscopic particles over space. Real-space coordinates are increasingly widely available, from electron microscopy studies [29] and simulations [30,31], to mesoscopic model systems such as colloidal dispersions [32,33], where optical microscopy can give the coordinates of all particles in bulk within a field of view [34]. There are advantages to the latter two techniques over electron microscopy techniques. First, 3D 
reconstruction of structure using electron microscopy once more requires serial sectioning which destroys the sample, preventing dynamic measurements; and second, electron microscopy is necessarily a surface technique meaning that surface effects must be ruled out from measurements.

With the availability of $3 \mathrm{D}$ coordinates, there is an increasing need for a robust means to extract the location and properties of grain boundaries from real-space data. However, existing grain and boundary detection schemes offer only a partial analysis. Some schemes use order parameters to locate grain boundaries [35,36] and by doing so neglect to measure orientation. Polyhedral template matching [37], used in the OVITO software [38], allows orientation measurement and grain resolution, but neglects grain boundary surfaces or local resolution of misorientations at the grain boundaries [39]. Many detection schemes are only useful in specific contexts, e.g., where a system is constrained to only one or one type of grain boundary and the orientation of one or more of the grains is known beforehand [31,32,40-43]. Taming grain boundaries like this is beneficial because it reduces their complexity. However, the resulting boundary properties only apply to themselves [16] because forces associated with ever-present neighboring grain boundaries are neglected.

Here, we present a generally applicable method to fully identify and characterize crystalline grains, crystalline structures, and to render grain boundaries using real-space particle coordinates. We apply the method to real-space coordinates of colloidal particles in dense crystalline dispersions. The method is shown to be robust against thermal noise as well as defects in the crystals, and to provide exhaustive information on both the location and orientation of several kinds of boundary. Importantly, we also provide a means to locally characterize the degree of misorientation between grains at different locations on the grain boundary surface, giving unprecedented access to the range of structures present. This paper is organized as follows. Section II details the grain and grain boundary characterization method. Section III then shows its application where we uncover unprecedented detail in the grain and grain boundary structure of both a templated colloidal bicrystal and a polycrystalline colloidal crystal from experimentally obtained particle coordinates.

\section{GRAIN AND GRAIN BOUNDARY CHARACTERIZATION METHOD}

To characterize grains and grain boundaries, we start with real-space coordinates of particles in a region of interest, and apply an analysis consisting of four steps: (i) the nearest neighbors of each particle are identified; (ii) the orientation and structure of each nearest-neighbor cluster is identified; (iii) grains are identified and separated; (iv) grain boundary locations and misorientations are found and visualized. The method is compatible with periodic boundary conditions, so may be directly applied to computational as well as experimental data.

\section{A. Nearest-neighbor identification}

Correct identification of the nearest-neighbor shell is essential for accurate structure and orientation assignment. For a given particle, the parent, we locate up to $N_{\max }$ nearest neigh- bors within a cutoff distance using a $k$ - $d$ tree [44] to determine neighbor distances. For close-packed monodisperse spheres, $N_{\max }=12$ due to geometrical constraints. The cutoff distance is typically the first minimum in the radial distribution function of the particle coordinates. Each "cluster" that is formed by a parent particle's nearest neighbors is referred to as the nearest-neighbor cluster (NNC). The number of particles in an NNC may be fewer than $N_{\max }$ to account for crystal vacancies.

\section{B. Registration, orientation, and structure assignment}

To identify the orientation and structure of each NNC, we require a quantitative comparison of experimentally observed specimen NNCs and a set of reference NNCs from perfect crystal structures. To do this, each point in a specimen NNC must be paired with their corresponding point in a reference NNC by aligning the two point sets. This process is known as point set registration and is a common procedure in computer vision processes [45]. Methods for point set registration that involve only translation and orientation transformations are called rigid registrations, which we will use here to dramatically simplify the process. This approach is valid because other small transformations have little effect on orientation measurements. In fact, the registration process can be further simplified by using the translational invariance property of crystal lattices. This is achieved by aligning the central point from the specimen NNC and the reference NNC. In doing so, the translation is removed from the registration calculation and the central parent points are registered. This simplification is valid for small displacements of the points from their lattice sites in the specimen NNC.

Point set registration is challenging because, to align two point sets, the corresponding pairs of points must be known, but to find the corresponding pairs of points the sets must be aligned. Only by chance will the reference and specimen sets align, and if we wish to calculate the orientations for many different crystallites, it is almost guaranteed that there will be some specimen sets that do not align with the reference set. There are two options for registration: (1) trials over orientations and (2) trials over corresponding pair permutations. Iterative methods are unreliable for NNC set alignment because there are many local maxima in the set alignment, where one point is aligned and others are not. Therefore, a registration scheme must span all possibilities to find the global maximum set alignment. Trials over orientations, considering option 1, requires a large number of trials for good angular resolution, which makes the computation prohibitively costly. It is important to have as small a number of trials as possible, particularly for large sets of real-space coordinates. For example, recent confocal microscopy studies have regions of interest containing in excess of 10000 particles (and therefore 10000 NNCs). Thus, we propose a method using option 2: trials over corresponding pair permutations. We increase the efficiency by significantly reducing the number of permutation trials.

The method proceeds as follows. Let the reference NNC and the specimen NNC have $N_{\mathrm{r}}$ and $N_{\mathrm{s}}$ elements, respectively. Note that the central parent points are registered from the translation step so they are not included. Set alignment is quantified by the sum of the $N_{\mathrm{s}}$ smallest distances between 
(a)

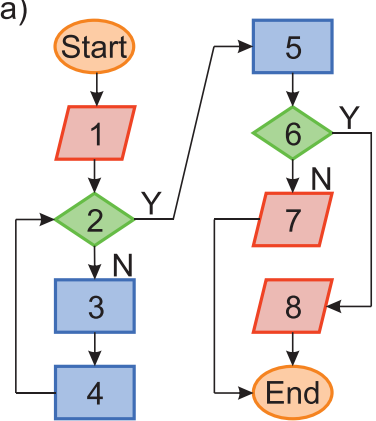

(b)

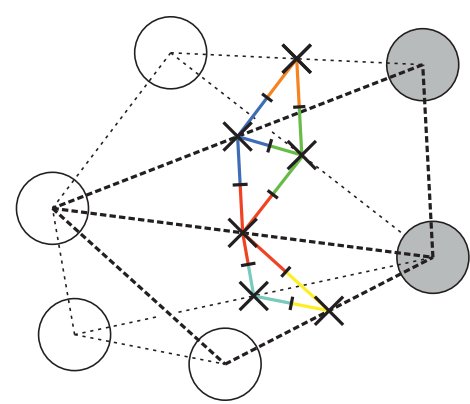

(c)

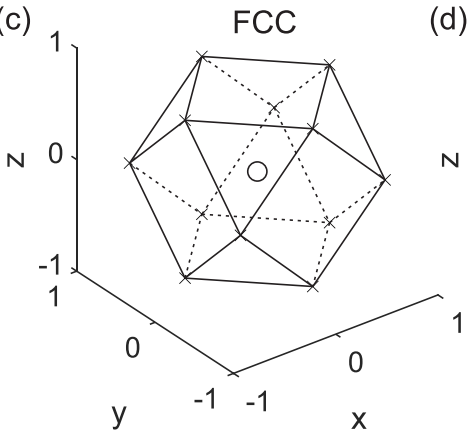

(d)

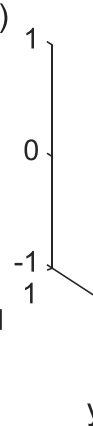

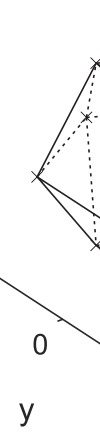

$\mathrm{HCP}$

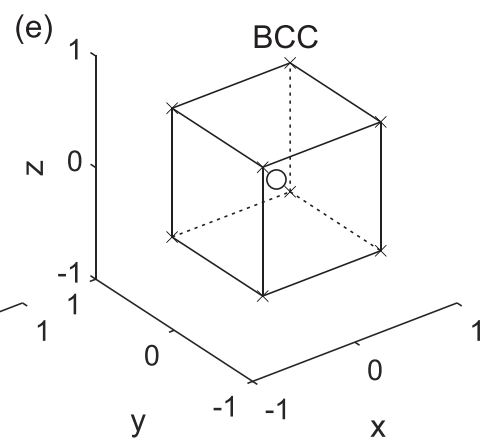

FIG. 1. (a) Flow chart of the point set registration process. Ovals denote the start or end, parallelograms are inputs or outputs, diamonds represent decisions, where $\mathrm{Y}$ and $\mathrm{N}$ stand for yes and no, respectively, and rectangles indicate processes. The detail for each number is given in the text. (b) Cartoon showing the grain boundary rendering process. The positions of particles in two different grains in three dimensions are shown by white and grey circles, respectively. They are not positioned in a crystalline arrangement for the purpose of clarity. Delaunay triangulation edges are shown as dotted lines (thickness for perspective) and grain boundary points are shown as black crosses. The grain boundary lines are shown in two colors, either side of a perpendicular bisector. (c), (d), and (e) show the NNCs for FCC, HCP and BCC, respectively. The black crosses mark the positions of particles and the black circle, the parent particle.

the point sets of the specimen NNC and the reference NNC. Maximum set alignment is achieved for the minimum value of this sum. A brute force approach would require trialing all permutations of pairs of points between the specimen and reference sets, equal to $N_{\mathrm{s}}$ !. For a close-packed crystal this is $12 ! \approx 4.8 \times 10^{8}$, which is prohibitively costly. However this number may be significantly reduced by taking advantage of the fact that for an orientation to be specified in $D$ dimensions it is enough to select only $D$ linearly independent vectors [46]. If the reference NNC and specimen NNC are related by a rigid transformation, as is the case for a perfect polycrystal where the reference and specimen NNC have the same structure, the sets can be aligned by considering only three corresponding pairs of points. Thus, in three dimensions, instead of $N_{\mathrm{s}}$ ! trials, the number is reduced to the permutations of three from $N_{\mathrm{r}}, N_{\mathrm{r}} P_{3}$, lowering the number of trials by a factor of $\left(N_{\mathrm{r}}-3\right)$ !. Therefore, corresponding pair trials are generated from the permutations of three points from the $N_{\mathrm{r}}$ reference NNC points paired with the same three randomly selected points from the specimen NNC. For a close-packed crystal, $\left(N_{\mathrm{r}}-3\right) !=9 ! \approx 10^{5}$, cutting the computational cost by five orders of magnitude. This number may be reduced further with consideration of the symmetry of the reference NNCs, as certain permutations of three particles from $N_{\mathrm{r}}$ are equivalent. For example, the number of trials required for face-centered cubic (FCC), hexagonal close-packed (HCP), and body centered cubic (BCC) NNCs reduces to 39, 223, and
17, respectively. A method to select symmetry in-equivalent permutations is given in Sec. I of the Supplemental Material [53].

The point set registration is laid out in the following sequence and illustrated in the flow diagram in Fig. 1(a) where $\mathrm{Y}$ and $\mathrm{N}$ represent yes and no, respectively.

(1) Input trials of in-equivalent permutations of three corresponding pairs.

(2) Is the number of completed loops equal to the number of trials of corresponding pairs? If no go to step 3 , if yes go to step 5.

(3) The next trial of three corresponding pairs is converted to an orientation by the Kabsch algorithm [47,48], which minimizes the squared distance between the pairs of corresponding points. Note we modify the Kabsch algorithm to fix the center of rotation to the parent particle. Each orientation is applied to all points in the specimen set in an attempt at set alignment.

(4) Calculate the sum of the $N_{\mathrm{S}}$ smallest distances between pairs of points, one from each NNC set. Return to step 2.

(5) The trial that produced an alignment with the minimum sum of the $N_{\mathrm{s}}$ smallest distances between pairs of points, one from each NNC set, establishes the corresponding pairs. Points are paired when the distance between them is one of the $N_{\mathrm{s}}$ smallest. The registration is formed by this set of pairs where every point in the specimen NNC has a corresponding point in the reference NNC. 
(6) Is any point paired with more than one other? If no go to step 7, if yes go to step 8. To enhance the accuracy of the method, registrations are only accepted if no point is paired with more than one other. This means that only specimen NNCs with a structure approximately equal to the reference are registered.

(7) Output accepted registration.

(8) Registration fails.

Although success in finding the global minimum in the sum of $N_{\mathrm{s}}$ smallest distances between the specimen and registered NNC points is only guaranteed for perfect polycrystals, this approach will still pair corresponding points in each set for specimen NNCs with small deviations from a perfect structure. The global minimum is found by using the accepted registration from step 7 , where corresponding pairs for all points in the specimen NNC are used (instead of just three) as the input for the Kabsch algorithm [47]. Again, we modify the Kabsch algorithm so the rotation center is fixed to the central parent particle, regardless of the center of mass of the NNC, to allow for robust orientation measurement when vacancies create incomplete NNCs. Furthermore, using all points ensures that deviations from perfect crystal positions in the specimen NNCs caused by thermal noise are accounted for. The reported orientation for each NNC is calculated from this complete registration.

Structure assignment is performed by minimizing the sum of the $N_{\mathrm{s}}$ smallest distances between corresponding pairs of points from the accepted registrations over reference NNC structures of interest. For example, a hard sphere system may have an FCC or HCP crystal structure, whereas a BCC crystal structure is expected for spheres with long-range repulsion. Therefore, one may wish to assign the set of references to contain three reference NNCs, one each for FCC, HCP, and BCC where $N_{\mathrm{r}}=12,12$, or 8 , respectively. The reference NNCs for FCC, HCP, and BCC are shown in Figs. 1(c), 1(d) and 1(e), respectively. The procedures in Sec. II.B can be parallelized over each specimen NNC individually for efficient computation.

\section{Separation of grains}

After the orientation of all NNCs have been measured, these may be used to separate different grains. First, a Delaunay triangulation between all parent particles with NNCs assigned the same structure forms a graph where each particle is a node and each pair of connected particles is a link. Since each link connects two different particles each with their own NNC orientation, a misorientation may be found. Like an orientation, a misorientation may be expressed as a rotation $\theta$ about an axis. Two thresholds are then applied to eliminate links and separate grains. The first is a distance cutoff $R_{\text {cut }}$ for NNCs with the same orientation, but separated in space. The second is $\theta_{\text {cut }}$, a maximum misorientation angle for adjacent NNCs in the same grain. This leaves disparate "clusters" of links between particles; each of these clusters form a single grain. A high $\theta_{\text {cut }}$ runs the risk of including particles that have intermediate orientation and position between grains, thus preventing grain separation. However, a low $\theta_{\text {cut }}$ may split grains to produce low-angle grain boundaries within. Particles with no neighbors within these thresholds are not assigned to a grain; they are not considered as a grain them- selves. As a result, the smallest grain possible consists of two particles. Note that this is much smaller than the typical size of critical crystal nuclei (hundreds of particles) $[49,50]$ demonstrating that this method may be useful in nucleation studies. This step also serves to confirm or reject the structure assigned in Sec. II B and suppresses the spurious identification of orientation and structure. Any particles not belonging to a grain are considered to have a structure different to the reference NNCs considered, which may include particles with a liquid-like local environment. Thus, this method also provides a way to distinguish crystalline from noncrystalline particles.

\section{Grain boundary location, misorientation, and visualisation}

Boundaries between grains may be resolved using a method similar to that used by Lavergne et al. [51], but extended to three dimensions. First, a Delaunay triangulation over particles belonging to a grain is calculated. The midpoints of Delaunay edges which connect particles in different grains form a surface of grain boundary points. Figure 1(b) shows a schematic of a triangulation of particles in two grains where the members of each grain are shown as white or grey circles, respectively. The dotted lines show the triangulation edges and the black crosses show the grain boundary points. Note that these points do not lie on a particle coordinate. Furthermore, a "local" misorientation may be calculated for each grain boundary point using the orientation of the particles on either end of the triangulation edge, yielding a spatial map of misorientations over the boundary surface. Grain boundary particles may be identified as those within a cutoff distance to a grain boundary point and not a member of a grain. In this way, grain boundary particles may be identified without searching for a particular arrangement of particles at the boundary, which is especially useful when searching for unstructured grain boundaries.

The geometry of the surface may be visualized in more detail by again considering the Delaunay traingulation. For every tetrahedron with vertices in two grains, the grain boundary points formed from its edges may be linked by lines to form edge-sharing triangles, as shown in Fig. 1(b). It is convenient to render the local misorientation by coloring the lines instead of the grain boundary points. This is done by bisecting each line and coloring each half by the misorientation of the closest grain boundary point. This can be seen in Fig. 1(b) where each line has two colors, one either side of the bisecting line. In this way grain boundaries are rendered as a triangle mesh where local misorientation information is represented by color. In this work we choose to map the color to the misorientation angle using an angle axis representation.

Note that the location of misorientations in threedimensional rotation space may be presented using vectorial representation. Here we use Rodigues-Frank vectors, $\overrightarrow{R F}=$ $\left(R F_{x}, R F_{y}, R F_{z}\right)$ within fundamental zones containing the smallest misorientation angle, within a standard stereographic traingle as defined in Ref. [52]. Rodrigues-Frank vectors are built from angle axis representation of rotations as $\overrightarrow{R F}=$ $\vec{A} \tan \left(\frac{\theta}{2}\right)$, where $\vec{A}$ is a normalized vector parallel to the rotation axis and $\theta$ is the rotation (misorientation) angle. 
(a)

(c)
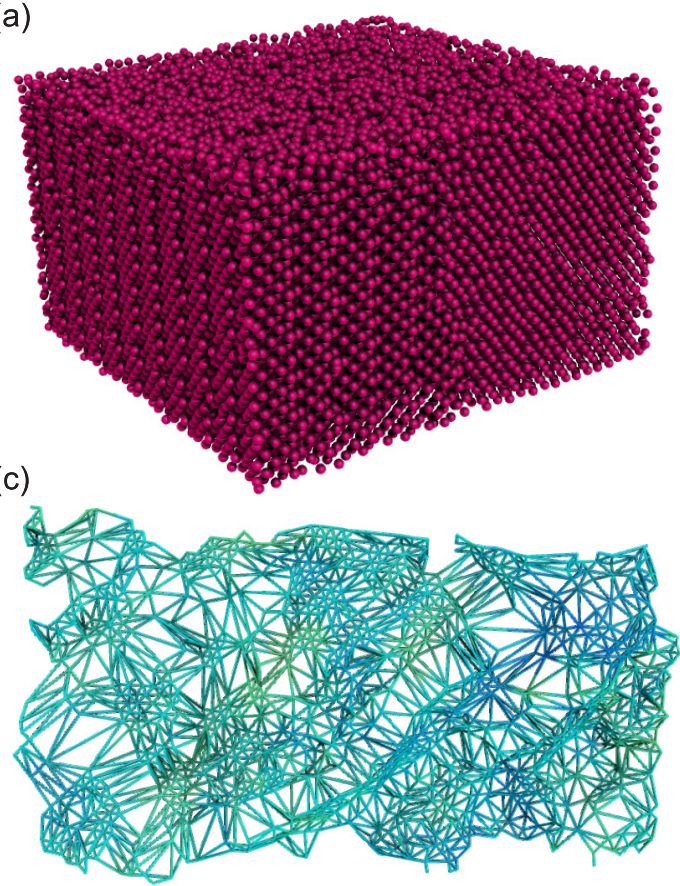

(b)

(d)
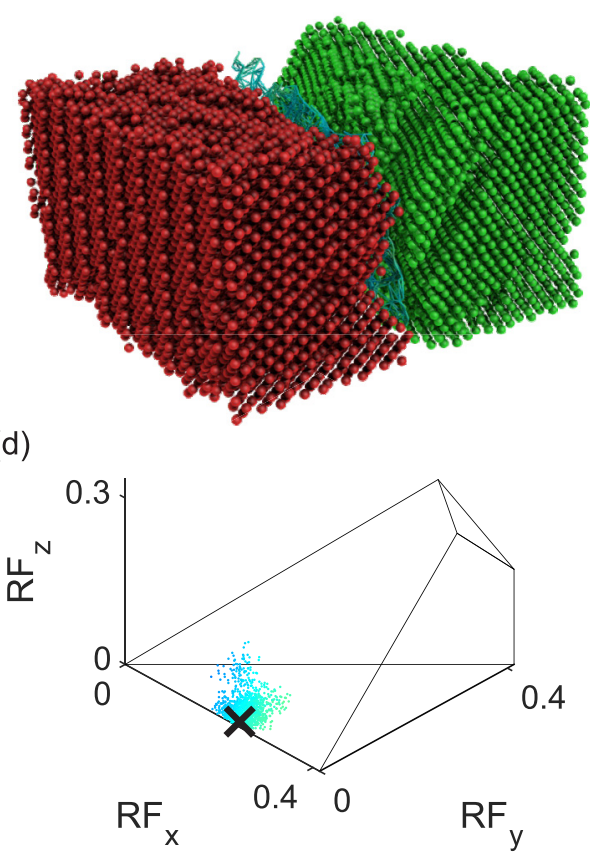

FIG. 2. Templated colloidal bicrystal. (a) Rendering of the colloidal crystal from particle coordinates. (b) Two largest FCC grains, either side of the $\Sigma 17$ boundary. (c) Normal projection of the $\Sigma 17$ boundary. (d) Rodrigues-Frank fundamental zone of misorientation containing the local misorientation for the boundary. The black cross shows the mean misorientation $\overrightarrow{R F}_{\mathrm{M}}=(0.2497,0.0088,0.0002)$.

\section{RESULTS AND DISCUSSION}

To demonstrate the utility and capabilities of this method, we study two colloidal crystals, a templated bicrystal using a patterned surface and a homogeneously nucleated polycrystal. We characterize misorientations of grain boundaries with particle level detail; one should note that singleparticle resolution is often presented as a key strength of colloidal models. Our method broadens this strength to grain boundary studies.

\section{A. Grain boundary structure in a templated colloidal bicrystal}

Here, we repurpose particle coordinates from a colloidal bicrystal produced to study grooves at grain-boundary-liquid triple junctions [32]. We use these data as a test case to demonstrate the method on a grain boundary of known location and misorientation. The sample was produced by slowly sedimenting a colloidal dispersion of monodisperse, $1.6 \mu \mathrm{m}$ diameter silica spheres onto a glass slide [32]. The glass slide was pretemplated using stereolithography, drilling dimples in positions corresponding to the first plane of a $\Sigma 17$ (a type of coincident site lattice) grain boundary between two compact planes. During slow sedimentation, the first layer of deposited particles sits in the dimples; subsequent layers grow by epitaxy by completing compact planes. The aqueous dispersion is index matched to allow deep penetration of the light without scattering; it also contains fluorescein so that the matrix can be easily distinguished from the nonfluorescent glass spherical particles. The sediment is imaged using 3D laser scanning confocal microscopy. Appropriate postprocessing finally allows the determination of the center of mass of each colloidal particle [32]. The cutoff distance for nearest neighbors is set to $2.0 \mu \mathrm{m}$, the first minimum of the radial distribution function, which sits at roughly 1.25 times the particle diameter. The distribution for the number of nearest neighbors is found in Sec. II of the Supplemental Material [53]. All NNCs with at least three neighbors of the parent particle were considered for orientation measurement, comparing against both FCC and HCP reference NNCs, which were scaled such that the average nearest-neighbor separation was $1.63 \mu \mathrm{m}$, the same as the first peak in the radial distribution function. The misorientation angle cutoff was set empirically to be $\theta_{\text {cut }}=2^{\circ}$, which is small enough to separate the grains in this data set. Section IV of the Supplemental Material [53] provides a discussion on the effects of different values of $\theta_{\text {cut }}$. The distance cutoff for grain boundaries was set to be the same as for nearest-neighbor detection, i.e., $R_{\text {cut }}=2.0 \mu \mathrm{m}$. The total computation time for the grains and grain boundaries in the following discussion from 38977 particle coordinates was $172 \mathrm{~s}$ using a quadcore Intel Core i5-6500 with 16 GB of RAM. The rendering was performed using open source PYMOL software [54].

Figure 2(a) shows a reconstruction of the coordinates of all particles. A boundary may be roughly identified by eye. Applying our method, the two largest FCC grains detected are shown in Fig. 2(b), colored by grain, sitting on either side of the nominal $\Sigma 17$ boundary. Initially, we consider only these two major grains, and identify a grain boundary between them. The misorientation is found to be $\overrightarrow{R F} \mathrm{M}=$ $(0.2497,0.0088,0.0002)$; in agreement with the value for a $\Sigma 17$ boundary in an FCC crystal, $\overrightarrow{R F} \Sigma 17=(0.25,0,0)$ [55]. Note that this average misorientation is calculated from the average orientation of each large FCC grain using an implementation [56] of the rotational averaging method 
described in Ref. [57]. If we render the geometry of the grain boundary surface and color code misorientations over the edges (see Sec. IID) as shown in Fig. 2(c), a broad spatial variation in misorientation is revealed. This is accordingly seen in the Rodrigues-Frank misorientation fundamental zone in Fig. 2(d), where points are scattered around the misorientation expected for a $\Sigma 17$ boundary, shown as a black cross. Thus, we show the method characterizes grain boundaries correctly and resolves spatial variation in misorientation at the particle level. Details of the grain boundary particles within $R_{\text {cut }}$ of the $\Sigma 17$ boundary may be found in Sec. III of the Supplemental Material [53]; the following grain boundary discussions will focus on the grain boundary surfaces, rather than the particles themselves.

Templating techniques such as those used to create this bicrystal [32] are designed to create a single boundary with a pre-engineered misorientation. However, the two major grains and the boundary between them do not account for all the grain and grain boundary structures present: this manufactured boundary is far more complex than a single surface with a single misorientation and contains both FCC and HCP crystal structures. Using our method we proceed to identify all FCC and HCP grains composed of 50 or more particles and characterize the multitude of unique boundaries which are present.

We first consider the range of FCC-FCC boundaries present in the system, in particular the twin boundaries. Twinning is of interest as particular twin geometries have consequences for grain growth kinetics [15]. To this end, HCP grains are omitted during the FCC-FCC grain boundary generation (as explained in Sec. IID) so that triangulation edges connect FCC grains on either side of a stacking fault. This prevents FCC twin boundaries being replaced by two grain boundaries between the two twinned FCC grains and the bisecting HCP layer. A particularly salient example of a twin boundary is shown in Fig. 3(a), where a smaller FCC grain, labeled $G_{\mathrm{t}}$ is surrounded by one of the major FCC grains. It is wrapped in an envelope-like grain boundary whose misorientation is located around $\overrightarrow{R F}=\left(\frac{1}{3}, \frac{1}{3}, \frac{1}{3}\right)$ in the FCC-FCC Rodrigues-Frank misorientation fundamental zone [see inset in Fig. 3(a)], as expected for a twin boundary in an FCC crystal. This boundary is composed of two different inclinations, one where the boundary plane has a normal perpendicular to the hexagonal plane direction, marked $i$ in Fig. 3(a), and another where the normal is contained in the hexagonal plane, marked ii. Here we see how the spatial resolution of our method reveals the fine details of grain boundaries. If we add the HCP stacking, we indeed find that the parts of the boundary parallel to the hexagonal planes, $i$, are coincident with single, isolated HCP layers. However, this is not case for the parts of the boundary that wrap around the small twin grain to connect these planes, $i$. In principle, one might also identify isolated FCC-HCP boundaries, as we will see below, but the ability to freely calculate the misorientation angle between grains of selected structure makes the detection of twin grain boundaries much simpler.

We proceed to consider the HCP crystalline regions and FCC-HCP boundaries. As shown in Fig. 3(b), the nominally "simple" $\Sigma 17$ boundary has a significant number of HCP layers extending into the FCC grains. The corresponding mis- orientations are plotted in the Rodrigues-Frank fundamental zone in Fig. 3(c) with a color depending on the misorientation angle $\theta$. Projecting the distribution of points in misorientation space onto a probability distribution in misorientation angle, $P(\theta)$ [see Fig. 3(d)], we see that there are multiple peaks. The distribution is magnified in the inset so that the peaks with smaller probabilities may be seen more clearly. The largest peak at misorientation angle $\theta \approx 56.6^{\circ}$ corresponds to Blackburn [58] boundaries between HCP and FCC grains either side of a stacking fault. The energy associated with stacking fault creation is very low so it is no surprise to see the highest probability at this position. Figure 3(c) shows the local misorientation distribution of these boundaries, scattered about the Blackburn position marked with an black cross at position $\overrightarrow{R F}$ B $=(0.414,0.318,0.132)$.

Note the presence of a smaller peak at $\theta \approx 49^{\circ}$, marked iii in Fig. 3(d). In fact, these correspond to the portion of the boundaries running approximately perpendicular to the hexagonal planes, which wrap around the HCP grains to connect two in-plane FCC-HCP boundaries. They are marked iii in Fig. 3(b), where they appear as a lighter shade of red. Their location in the Rodrigues-Frank misorientation fundamental zone is also marked iii in Fig. 3(c). Figure 3(e) shows how particle positions vary across this FCC-HCP boundary, where the boundary adopts this lower misorientation angle. There is no abrupt change from HCP to FCC; instead, the hexagonal planes gradually shift relative to one another. This results in NNCs in the vicinity of the boundary being distorted. Here, NNC particles are displaced in a concerted manner with their counterparts from the same hexagonal plane, but differently from members in other hexagonal planes. This results in a net rotation of the NNCs near the boundary. Note that the Blackburn boundaries have the maximum misorientation possible, i.e., the full misorientation between the two structures. Thus, the misorientation angle is necessarily reduced for the distorted NNCs. This kind of boundary has been identified before in colloidal crystals by Hilhorst et al. [59], although without a specific detection scheme.

Having located both FCC-FCC and FCC-HCP boundaries over all grains in the system, we are now in a position to characterize the $\Sigma 17$ boundary in more depth. The "single" boundary identified in Fig. 2(c) using the two major FCC grains now looks very different [see Fig. 3(f)]. The blue portion marked as $\Sigma 17$ corresponds to FCC-FCC boundaries, nominally the "remnants" of the original $\Sigma 17$ boundary. However, there are now other contributions including FCCHCP boundaries with different misorientation where the HCP grains meet FCC grains at the boundary. These are marked $i v$ and shown in yellow; they also appear as surfaces in Fig. 3(b) and in the misorientation distribution Fig. 3(c). The misorientation angles of these boundaries peak at $\theta \approx$ $39^{\circ}$, marked $i v$ in the misorientation angle distribution in Fig. 3(d).

Another contribution to the boundary in Fig. 3(f) is the FCC-HCP boundary between the twin grain $G_{\mathrm{t}}$ from Fig. 3(a) and HCP layers on the other side of the $\Sigma 17$ plane. This is shown in green on the left-hand side and marked $v$. This is also visible at peak $v$ at $\theta \approx 30^{\circ}$ in Fig. 3(d). Finally, there is a contribution from the junction between $G_{\mathrm{t}}$ and the major FCC grain on the other side of the $\Sigma 17$ plane, marked $v i$. 
(a)

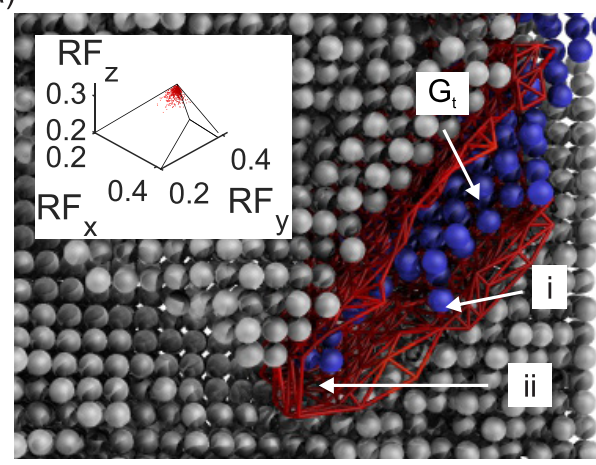

(c)

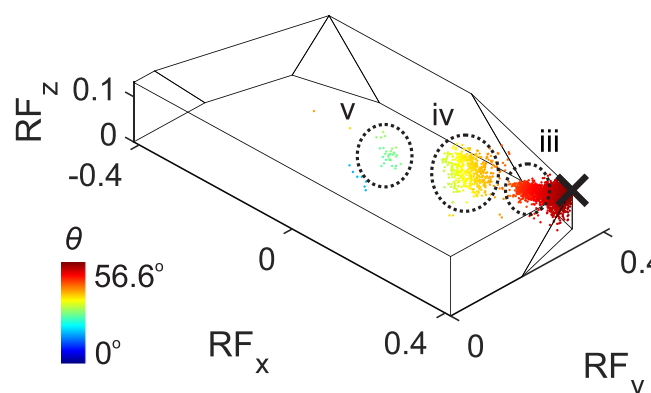

(b)

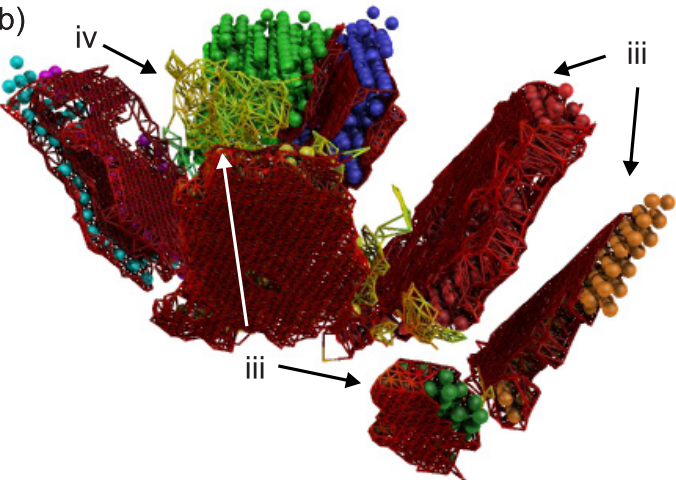

(d)

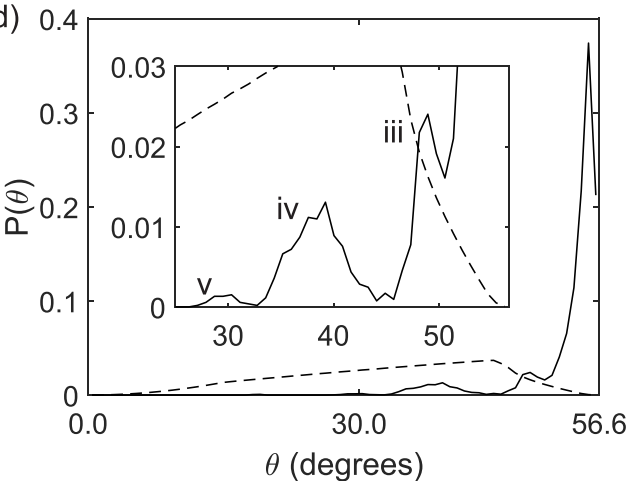

(e)

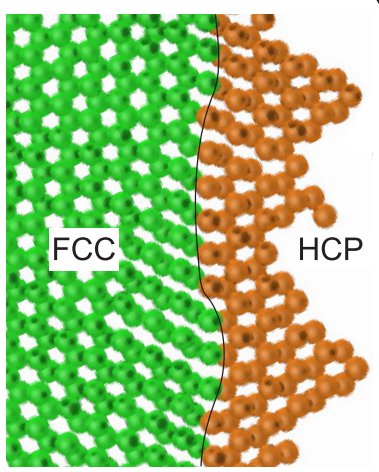

(f)

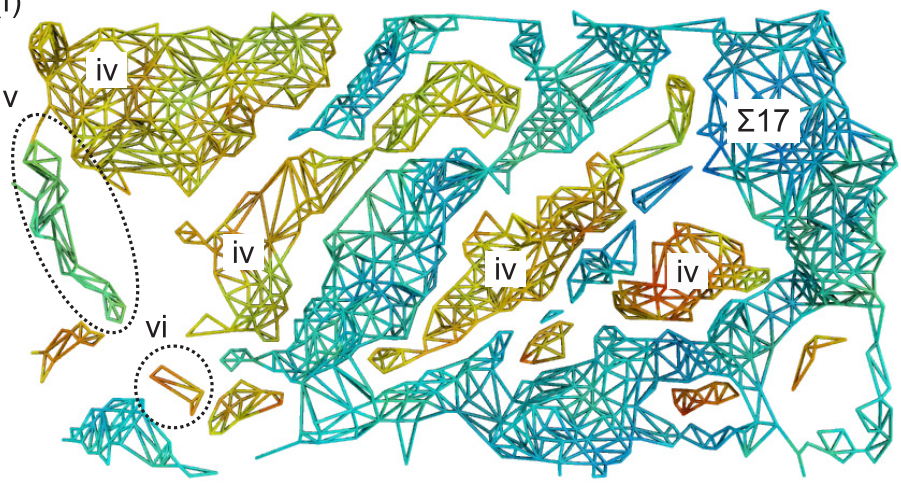

FIG. 3. A selection of other grain and grain boundary structures within the templated colloidal bicrystal, the numbered labels, $i$ through $v$, included in several panels are explained in the main text. (a) FCC twin boundary and twin grain $G_{\mathrm{t}}$. The inset shows the local misorientation distribution. (b) HCP grains and FCC-HCP grain boundaries. The particles are colored by grain identity and the grain boundaries by local misorientation angle $\theta$ [see color bar in (c)]. (c) Rodrigues-Frank misorientation distribution within the fundamental zone for boundaries between FCC and HCP grains, colored by misorientation angle (see color bar). (d) Local misorientation angle distribution for FCC-HCP boundaries. The inset shows a magnified portion. (e) In-plane stacking sequence change from FCC to HCP. The black line is a visual guide. (f) A mosaic of grain boundary structures including the $\Sigma 17$ boundary and the contributions of other boundaries.

With our method we characterized the grain and grain boundary structure of a pre-templated $\Sigma 17$ boundary [32] in great detail. Despite the misorientation between the major grains being as designed, the microstructure of the boundary itself is far more complex, with a combination of defects and highly curved grain boundaries with misorientations that do not exactly conform to the angles predicted for simple stacking faults. Furthermore, the difference in structure of the boundary can contribute to a difference in the properties, which may go unexamined without particle level structural characterization.

\section{B. Characterization of homogeneously nucleated polycrystalline structures}

We extend the analysis to a sample where we have no preknowledge of the position or misorientation of the grain boundaries. We demonstrate this by analyzing a homogeneously nucleated colloidal polycrystal. The finer grain structure showcases the spatial resolution of our method for grain and grain boundary detection. We apply the algorithm to coordinates of particles in a polycrystalline colloidal sample composed of $\approx 2.4 \mu \mathrm{m}, 3 \%$ polydisperse, polydimethylsiloxane stabilized core-shell PMMA spheres. 
The synthesis of the particles is based on the methods presented in Refs. [60,61]. The particles are suspended in a density and index matching mixture of cyclohexyl bromide and cis-decahydronaphthalene saturated with tetrabutylammonium bromide (TBAB) to achieve electrostatic screening. The particles are dyed with trace amounts of Cyanine 3 in the particle core for laser scanning confocal microscopy imaging with a Leica SP5 scanhead. The particle coordinates are recovered from the confocal images using conventional particle identification software [34].

To prevent heterogeneous nucleation, the glass walls of the sample cell were coated with polydisperse colloidal PMMA particles to create a uniform but rough surface on the length scale of the colloidal particle. To this end, a $22 \times 22 \mathrm{~mm}$ number 1 cover slip was spin coated with a dense, polydisperse suspension of PMMA particles (20\% volume fraction, $30 \%$ polydispersity in size) in cis-decahydronaphthalene, taking care to avoid bulges on the outer rim of the cover slip. This is immediately transferred to a $120^{\circ} \mathrm{C}$ hot plate to dry the solution rapidly and avoid de-wetting. This cover slip forms the imaging wall of a glass sample cell. A small glass bottle with the bottom removed is attached to the cell to allow the placement of the colloidal dispersion via pipette. The cell is sealed by attaching screw top lid with a teflon liner to the bottle top. The colloidal sample is left for several hours to crystallize, after which a field of view far away from the flat walls of the container is investigated.

As in the case of the templated bicrystal, the nearestneighbor and grain boundary cutoff distances are set to equal the first minimum of the radial distribution function, in this instance, $R_{\text {cut }}=3.0 \mu \mathrm{m}$ (once more roughly 1.25 times the particle diameter), and the reference NNCs are scaled to have a nearest-neighbor distance equal to the first peak of the radial distribution function $(2.35 \mu \mathrm{m})$. The distribution of numbers of nearest neighbors for the particles is given in Sec. II of the Supplemental Material [53]. The misorientation angle cutoff is set to $\theta_{\text {cut }}=2^{\circ}$. However, this time we do not impose a cutoff for the size of grains; thus, the smallest grains can now consist of as few as two NNCs. The total computation time was $234 \mathrm{~s}$ for 44025 particle coordinates on the same quadcore Intel Core i5-6500 with 16 GB of RAM.

Figures 4(a) and 4(b) show the FCC and HCP grains, respectively, where each grain is assigned a unique color. Some colors may appear indistinguishable because there are many colors for the many grains: 866 FCC grains and 964 HCP grains, with grain sizes range from 2 to approximately 500 particles. The number of particles in FCC and HCP grains, respectively, are 8606 and 5368, showing a random hexagonal close-packed structure with a slight preference for FCC and, in general, HCP grains that are smaller than FCC grains. A striking feature of both Figs. 4(a) and 4(b) are the planar gaps, and flat, narrow grains. These are formed by faults, where FCC changes to HCP or vice versa and serve to break up the crystallites parallel to the hexagonal layers. We may subsequently identify FCC-FCC, FCC-HCP, and HCP-HCP boundaries. Each row of Fig. 4 from Fig. 4(c) to 4(e) contains three panels that present from left to right the location of grain boundaries, the misorientation within the fundamental zone, and a misorientation angle distribution, respectively. As before, grain boundaries and their distributions are colored by their misorientation angle $\theta$ and the colors are consistent for each row. The color maps used to label the boundary points in Figs. 4(c) to 4(e) in both real and misorientation space span angles between $0^{\circ}$ and the maximum misorientation angle.

Again, we start by looking at FCC-FCC boundaries, omitting the HCP grains [see Fig. 4(c)]. The boundary structure is dominated by low angle and twin grain boundaries, in approximately equal measure. They occur where an even or odd number of HCP stacked hexagonal layers, respectively, separate two FCC grains. Accordingly, these boundaries are flat and lie parallel to the local hexagonal plane direction as seen in Fig. $4\left(\mathrm{c}_{1}\right)$. The corresponding misorientation distribution is shown in Fig. 4( $\left.c_{2}\right)$ where the misorientations at low angle and twin boundaries are concentrated around the points $\overrightarrow{R F}_{0}=(0,0,0)$ and $\overrightarrow{R F} \mathrm{~T}=\left(\frac{1}{3}, \frac{1}{3}, \frac{1}{3}\right)$, respectively, in the Rodrigues-Frank fundamental zone. Accordingly, these boundaries appear as peaks in the misorientation angle distribution in Fig. $4\left(\mathrm{c}_{3}\right)$ at $\theta \approx 0^{\circ}$ and $\theta \approx 60^{\circ}$. The peak at $\theta \approx 0^{\circ}$ and the twin peak at $\theta \approx 60^{\circ}$ have approximately equal probabilities relative to the random misorientation distribution, showing that stacking faults contain an equal proportion of even and odd hexagonally close-packed layers. Note that the form of the misorientation distribution highlights the singleparticle resolution, resolving stacking faults that separate FCC grains of the same orientation and that separate twin FCC grains. Note that all these twin boundaries are planes, unlike the example shown in the templated bicrystal where an FCC-FCC boundary bends around to encapsulate an FCC crystallite as in Fig. 3(a). None of the grains in this sample are large enough to support the in-plane stacking fault required to generate such a boundary. Figures $4\left(c_{2}\right)$ and $4\left(c_{3}\right)$ show that there are also a significant proportion of misorientations located between the peaks at $\theta \approx 60^{\circ}$ and $\theta \approx 0^{\circ}$. These misorientations are a hallmark of a polycrystalline sample, displaying many differently oriented grains. The surface area of these boundaries is far smaller than the twin and low angle boundaries so they appear with lower probabilities.

We may also look at HCP-HCP boundaries by omitting the FCC grains [see Fig. 4(d)]. Again, the structure is dominated by boundaries arising from stacking sequence changes of hexagonal planes. The most probable peak appears at $\theta \approx 0^{\circ}$ in Fig. $4\left(\mathrm{~d}_{3}\right)$ and the corresponding boundaries are shown in blue in Figs. $4\left(d_{1}\right)$ and $4\left(d_{2}\right)$. These boundaries are created where one or more FCC layers intersect two HCP grains, exposing a gap between two HCP grains of the same orientation. The other dominating feature of the distribution in Fig. $4\left(\mathrm{~d}_{3}\right)$ is at $\theta \approx 70^{\circ}$. This is again a consequence of stacking sequence changes, only this time along different hexagonal plane normal directions. Specifically, they are the different hexagonal plane normal directions in the FCC structure. The angle between these planes is equal to $70.53^{\circ}$ and accordingly, boundaries with misorientation $\theta \approx 70^{\circ}$ appear with a significant probability.

Finally, considering FCC-HCP boundaries [see Fig. 4(e)], we see a grain boundary structure dominated by the Blackburn misorientation relationship, corresponding to very high stacking disorder. Again, this is evidence for the random close-packing structure of the polycrystal. Figure $4\left(\mathrm{e}_{1}\right)$ shows mostly planar boundaries coloured in red corresponding to a Blackburn misorientation of $\overrightarrow{R F} F_{\mathrm{B}}=(0.414,0.318,0.132)$ as 


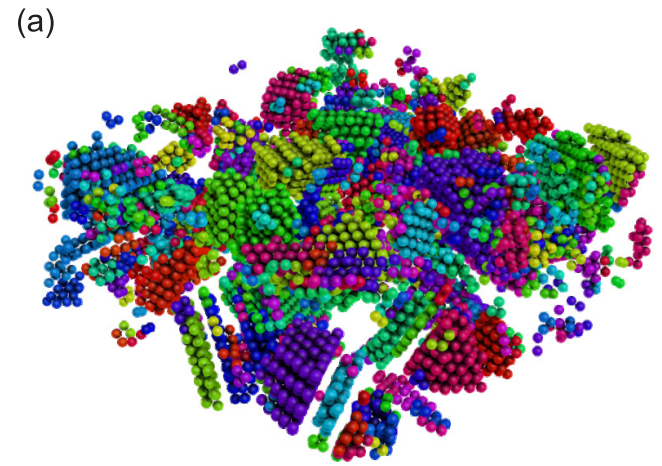

(c)

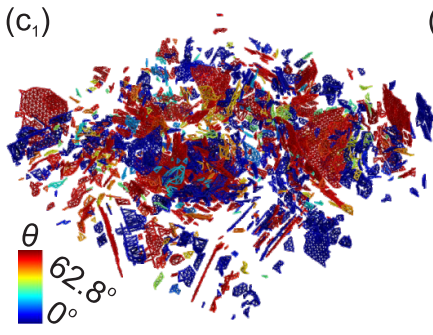

$\left(d_{1}\right)$

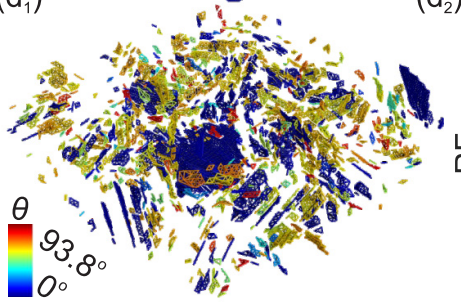

$\left(e_{1}\right)$

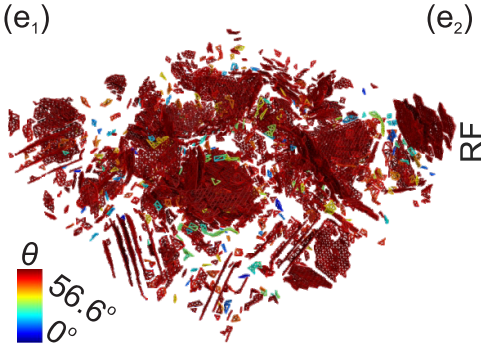

$\left(e_{2}\right)$ $\left(c_{2}\right)$

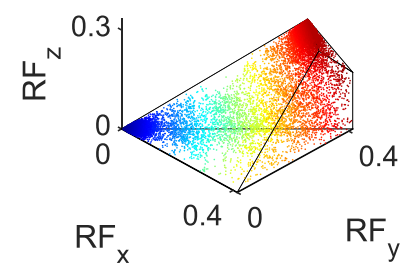

(b)

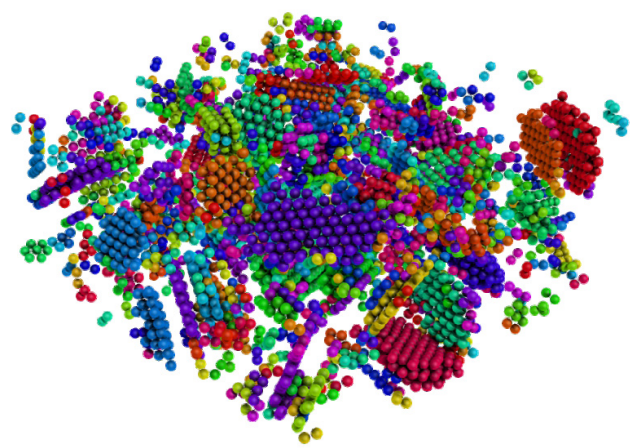

(c)

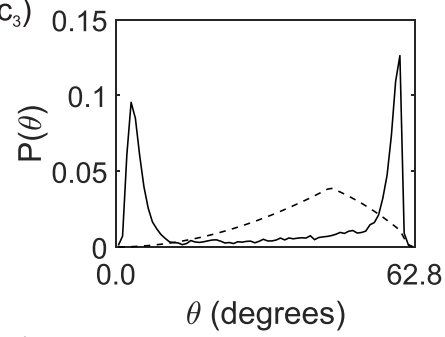

$\left(d_{3}\right)$

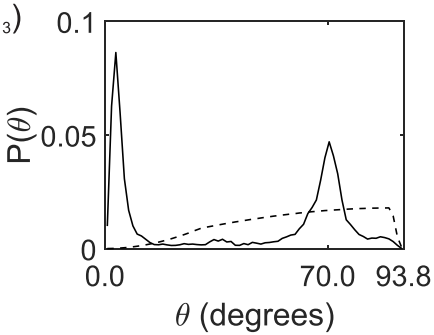

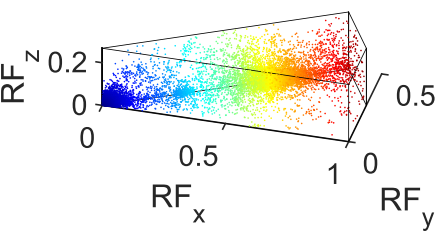

$\left(e_{3}\right)$
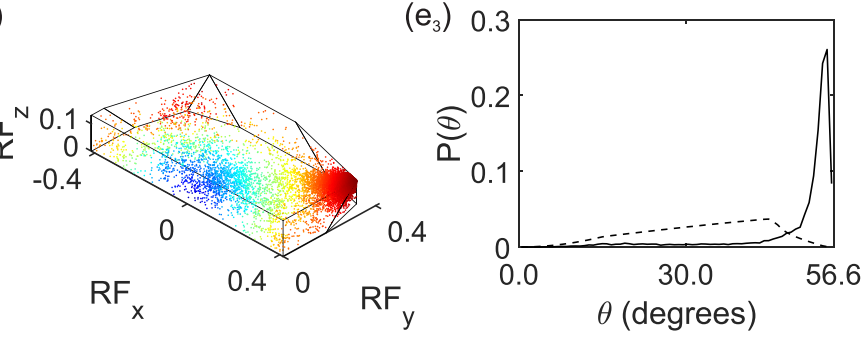

FIG. 4. The grain and grain boundary structures within the colloidal polycrystal. (a) and (b) show FCC grains and HCP grains, respectively. Each grain is given a unique color. The remaining panels of the image are organised as follows. Rows (c), (d), and (e) show characterization for grain boundaries between FCC grains omitting HCP grains, between HCP grains omitting FCC grains, and between FCC and HCP grains, respectively. Column (1) shows the spatial distribution of grain boundaries. Column (2) shows the misorientation distribution in the Rodrigues-Frank fundamental zone. Column (3) contains probability distributions of misorientation angles. The dotted lines show distributions for randomly oriented grains. The color bar extends from the smallest rotation angle to the largest and governs the colors for the panels in the same row.

shown in Fig. 4(e $\left.e_{2}\right)$. The distribution has a proportionately large peak at $\theta \approx 56^{\circ}$ in Fig. 4( $\left.\mathrm{e}_{3}\right)$. However, there is now no small peak at $\theta \approx 49^{\circ}$ due to the absence of in-plane stacking faults. These grains are smaller than in the bicrystal discussed in Sec. III A. Much like the absence of any curved FCC-FCC twin boundaries in the polycrystalline sample there is also an absence of curved FCC-HCP boundaries. In the templated bicrystal of Sec. III A, the stacking changes over roughly six particle spacings as shown in Fig. 3(f), similar in lengthscale to the in-plane stacking faults of Hilhorst et al. [59]; this is too large a lengthscale for the finer grains of the polycrystal. Hence, no boundaries due to in-plane stacking faults are seen in either FCC-HCP boundaries or indeed FCC-FCC boundaries as presented earlier.

\section{CONCLUSION}

A complete method is presented to convert real-space particle coordinates of polycrystalline materials to the location and character of grains and grain boundaries. Heterogeneity in misorientations may be characterized with single-particle resolution over boundaries without a priori knowledge of local crystal structure, giving unprecedented insight into a wide range of faults and defects, and how the degree of 
misorientation might affect physical properties. Here, we apply this to colloidal crystals, finding an unexpected degree of structural complexity in a nominally "tame" [16] templated system and how the distribution of misorientations change when similar particles are instead allowed to homogeneously nucleate crystals and grow. Importantly, we identify features such as twin boundaries, the Blackburn orientation relationship and low angle grain boundaries without targeting different portions of the structure. Our method may be applied to both experimental and numerical data with minimal knowledge of crystal orientations in a region of interest and would serve as a useful tool in future studies of polycrystals, nucleation, and grain growth. The routines are freely available [62] to other users.

\section{ACKNOWLEDGMENTS}

We acknowledge F. Spaepen for facilitating the acquisition of the colloidal bicrystal data shown in Sec. III A. We also acknowledge F. A. Lavergne, A. E. Stones, P. J. Lu, and H. Tanaka for insightful discussions and comments. The ERC (ERC Consolidator Grant No. 724834 OMCIDC) is acknowledged for financial support.
[1] Z. W. Shan, E. A. Stach, J. M. K. Wiezorek, J. A. Knapp, D. M. Follstaedt, and S. X. Mao, Grain boundary-mediated plasticity in nanocrystalline nickel, Science 305, 654 (2004).

[2] A. M. Alsayed, M. F. Islam, J. Zhang, P. J. Collings, and A. G. Yodh, Premelting at defects within bulk colloidal crystals, Science 309, 1207 (2005).

[3] R. Lipowsky, Melting at Grain Boundaries and Surfaces, Phys. Rev. Lett. 57, 2876 (1986).

[4] J. Q. Broughton and G. H. Gilmer, Thermodynamic Criteria for Grain Boundary Melting - A Molecular Dynamics Study, Phys. Rev. Lett. 56, 2692 (1986).

[5] H. Hilgenkamp and J. Mannhart, Grain boundaries in high- $T_{c}$ superconductors, Rev. Mod. Phys. 74, 485 (2002).

[6] S. Gokhale, K. H. Nagamanasa, R. Ganapathy, and A. K. Sood, Grain growth and grain boundary dynamics in colloidal polycrystals, Soft Matter 9, 6634 (2013).

[7] K. H. Nagamanasa, S. Gokhale, R. Ganapathy, and A. K. Sood, Confined glassy dynamics at grain boundaries in colloidal crystals, Proc. Natl. Acad. Sci. 108, 11323 (2011).

[8] E. O. Hall, The deformation and ageing of mild steel: III Discussion of results, Proc. Phys. Soc. B 64, 747 (1951).

[9] N. J. Petch, The cleavage strength of polycrystals, J. Iron Steel Inst., London 174, 25 (1953).

[10] K. Busch and S. John, Photonic band gap formation in certain self-organizing systems, Phys. Rev. E 58, 3896 (1998).

[11] G. A. Ozin and S. M. Yang, The Race for the Photonic Chip: Colloidal Crystal Assembly in Silicon Wafers, Adv. Funct. Mater. 11, 95 (2001).

[12] K. S. Napolskii, N. A. Sapoletova, D. F. Gorozhankin, A. A. Eliseev, D. Y. Chernyshov, D. V. Byelov, N. A. Grigoryeva, A. A. Mistonov, W. G. Bouwman, K. O. Kvashnina, A. V. Lukashin, A. A. Snigirev, A. V. Vassilieva, S. V. Grigoriev, and A. V. Petukhov, Fabrication of artificial opals by electric-fieldassisted vertical deposition, Langmuir 26, 2346 (2010).

[13] A. P. Sutton, E. P. Banks, and A. R. Warwick, The fivedimensional parameter space of grain boundaries, Proc. R. Soc. A. 471, 20150442 (2015).

[14] T. J. Rupert, D. S. Gianola, Y. Gan, and K. J. Hemker, Experimental observations of stress-driven grain boundary migration, Science 326, 1686 (2009).

[15] S. L. Thomas, A. H. King, and D. J. Srolovitz, When twins collide: Twin junctions in nanocrystalline nickel, Acta Mater. 113, 301 (2016).
[16] J. Han, S. L. Thomas, and D. J. Srolovitz, Grain-boundary kinetics: A unified approach, Prog. Mater. Sci. 98, 386 (2018).

[17] G. Gottstien and L. S. Shvindlerman, Grain Boundary Migration in Metals (CRC Press, Boca Raton, FL, 2009), pp. 513-533.

[18] B. L. Adams, S. I. Wright, and K. Kunze, Orientation imaging - the emergence of a new microscopy, Metall. Trans. A 24, 819 (1993).

[19] B. L. Adams, Orientation imaging microscopy: Emerging and future applications, Ultramicroscopy 67, 11 (1997).

[20] G. Johnson, A. King, M. G. Honnicke, J. Marrow, and W. Ludwig, X-ray diffraction contrast tomography: A novel technique for three-dimensional grain mapping of polycrystals. II. The combined case, J. Appl. Crystallogr. 41, 310 (2008).

[21] W. Ludwig, S. Schmidt, E. Mejdal Lauridsen, and H. F. Poulsen, X-ray diffraction contrast tomography: A novel technique for three-dimensional grain mapping of polycrystals. I. Direct beam case, J. Appl. Crystallogr. 41, 302 (2008).

[22] M. P. Echlin, N. S. Husseini, J. A. Nees, and T. M. Pollock, A New Femtosecond Laser-Based Tomography Technique for Multiphase Materials, Adv. Mater. 23, 2339 (2011).

[23] P. Reischig, A. King, L. Nervo, N. Vigano, Y. Guilhem, W. J. Palenstijn, K. J. Batenburg, M. Preuss, and W. Ludwig, Advances in X-ray diffraction contrast tomography: Flexibility in the setup geometry and application to multiphase materials, J. Appl. Crystallogr. 46, 297 (2013).

[24] M. D. Uchic, M. A. Groeber, D. M. Dimiduk, and J. P. Simmons, 3D microstructural characterization of nickel superalloys via serial-sectioning using a dual beam FIB-SEM, Scr. Mater. 55, 23 (2006).

[25] N. Bozzolo, L. Chan, and A. D. Rollett, Misorientations induced by deformation twinning in titanium, J. Appl. Crystallogr. 43, 596 (2010).

[26] J. M. Dake, J. Oddershede, H. O. Sørensen, T. Werz, J. C. Shatto, K. Uesugi, S. Schmidt, C. E. Krill Iii, C. E. K. Performed, and C. E. K. Ana-Lyzed, Direct observation of grain rotations during coarsening of a semisolid $\mathrm{Al}-\mathrm{Cu}$ alloy, Proc. Natl. Acad. Sci. 113, E5998 (2016).

[27] A. King, P. Reischig, S. Martin, J. F. B. D. Fonseca, and M. E. A. Preuss, Grain mapping by diffraction contrast tomography: Extending the technique to the sub-grain information, in Proceedings of the 31st Ris $\phi$ International Symposium on "Challenges in Materials Science and Possibilities in 3D and 4D Characterization Techniques”, edited by N. Hansen, 
D. Juul Jensen, S. F. Nielsen, H. F. Poulsen, and B. Ralph (Risø National Laboratory, Roskilde, Denmark, 2010).

[28] H. F. Poulsen, An introduction to three-dimensional X-ray diffraction microscopy 1, J. Appl. Crystallogr. 45, 1084 (2012).

[29] G. Lucadamo and D. L. Medlin, Geometric origin of hexagonal close packing at a grain boundary in gold, Science 300, 1272 (2003).

[30] C. P. Race, J. von Pezold, and J. Neugebauer, Role of the mesoscale in migration kinetics of flat grain boundaries, Phys. Rev. B 89, 214110 (2014).

[31] C. P. Race, R. Hadian, J. von Pezold, B. Grabowski, and J. Neugebauer, Mechanisms and kinetics of the migration of grain boundaries containing extended defects, Phys. Rev. B 92, 174115 (2015).

[32] E. Maire, E. Redston, M. Persson Gulda, D. A. Weitz, and F. Spaepen, Imaging grain boundary grooves in hard-sphere colloidal bicrystals, Phys. Rev. E 94, 042604 (2016).

[33] Y. Liu, K. V. Edmond, A. Curran, C. Bryant, B. Peng, D. G. A. L. Aarts, S. Sacanna, and R. P. A. Dullens, CoreShell Particles for Simultaneous 3D Imaging and Optical Tweezing in Dense Colloidal Materials, Adv. Mater. 28, 8001 (2016).

[34] J. C. Crocker and D. G. Grier, Methods of digital video microscopy for colloidal studies, J. Colloid Interface Sci. 179, 298 (1996).

[35] P. J. Steinhardt, D. R. Nelson, and M. Ronchetti, Bondorientational order in liquids and glasses, Phys. Rev. B 28, 784 (1983).

[36] E. A. Padston, Structure and dynamics of colloidal grain boundaries, Ph.D. Thesis, Harvard University, 2018.

[37] P. M. Larsen, S. Schmidt, and J. Schiøtz, Robust structural identification via polyhedral template matching, Model. Simul. Mater. Sci. Eng. 24, 055007 (2016).

[38] A. Stukowski, Visualization and analysis of atomistic simulation data with OVITO - the open visualization tool, Model. Simul. Mater. Sci. Eng. 18, 015012 (2010).

[39] M. Wagih and C. A. Schuh, Spectrum of grain boundary segregation energies in a polycrystal, Acta Mater. 181, 228 (2019).

[40] A. Van Blaaderen, R. Ruel, and P. Wiltzius, Template-directed colloidal crystallization, Nature (London) 385, 321 (1997).

[41] J. L. Priedeman, C. W. Rosenbrock, O. K. Johnson, and E. R. Homer, Quantifying and connecting atomic and crystallographic grain boundary structure using local environment representation and dimensionality reduction techniques, Acta Mater. 161, 431 (2018).

[42] T. Frolov, D. L. Olmsted, M. Asta, and Y. Mishin, Structural phase transformations in metallic grain boundaries, Nat. Commun. 4, 1899 (2013).

[43] A. Yamanaka, K. McReynolds, and P. W. Voorhees, Phase field crystal simulation of grain boundary motion, grain rotation and dislocation reactions in a BCC bicrystal, Acta Mater. 133, 160 (2017).

[44] J. L. Bentley, Multidimensional binary search trees used for associative searching, Commun. ACM 18, 509 (1975).
[45] L. Cheng, S. Chen, X. Liu, H. Xu, Y. Wu, M. Li, and Y. Chen, Registration of laser scanning point clouds: A review, Sensors 18, 1641 (2018).

[46] A. Morawiec, Orientations and Rotations (Springer, New York, 2004).

[47] W. Kabsch, A solution for the best rotation to relate two sets of vectors, Acta Crystallogr. A32, 922 (1976).

[48] E. Schreiber, Kabsch algorithm (https://www.mathworks.com/ matlabcentral/fileexchange/25746-kabsch-algorithm), MATLAB Central File Exchange. [Retrieved January 11, 2018], version 1.18.0.0.

[49] F. Leoni and J. Russo, Nonclassical Nucleation Pathways in Stacking-Disordered Crystals, Phys. Rev. X 11, 031006 (2021).

[50] P. R. ten Wolde, M. J. RuizMontero, and D. Frenkel, Numerical calculation of the rate of crystal nucleation in a LennardJones system at moderate undercooling, J. Chem. Phys. 104, 9932 (1996).

[51] F. A. Lavergne, D. G. A. L. Aarts, and R. P. A. Dullens, Anomalous Grain Growth in a Polycrystalline Monolayer of Colloidal Hard Spheres, Phys. Rev. X 7, 041064 (2017).

[52] A. Heinz, P. Neumann, and IUCr, Representation of orientation and disorientation data for cubic, hexagonal, tetragonal and orthorhombic crystals, Acta Crystallogr., Sect. A: Found. Crystallogr. 47, 780 (1991).

[53] See Supplemental Material at http://link.aps.org/supplemental/ 10.1103/PhysRevMaterials.5.123605 for a description of how symmetry inequivalent points are selected from the set of points in the reference nearest-neighbor cluster, NNC. We also show the number of particles within NNCs from both data sets. Additionally, we discuss how grain boundary particles may be identified and we provide an example. Finally, we explain how the misorientation angle cutoff threshold effects the misorientation distribution and grain boundary identification.

[54] The PYMOL Molecular Graphics System, Version 2.4, Schrödinger, LLC, (2020).

[55] H. Grimmer, W. Bollmann, and D. Warrington, Coincidencesite lattices and complete pattern-shift in cubic crystals, Acta Crystallogr. Sect. A 30, 197 (1974).

[56] T. Birdal, averaging_quaternions (https://github.com/ tolgabirdal/averaging_quaternions) [Retrieved July 15, 2021].

[57] F. L. Markley, Y. Cheng, J. L. Crassidis, and Y. Oshman, Averaging Quaternions, J. Guid. Control. Dyn. 30, 1193 (2007).

[58] R. Krakow, R. J. Bennett, D. N. Johnstone, Z. Vukmanovic, W. Solano-Alvarez, S. J. Lainé, J. F. Einsle, P. A. Midgley, C. M. F. Rae, and R. Hielscher, On three-dimensional misorientation spaces, Proc. R. Soc. A 473, 20170274 (2017).

[59] J. Hilhorst and A. V. Petukhov, Variable Dislocation Widths in Colloidal Crystals of Soft Thermosensitive Spheres, Phys. Rev. Lett. 107, 095501 (2011).

[60] R. P. A. Dullens, M. Claesson, D. Derks, A. Van Blaaderen, and W. K. Kegel, Monodisperse core-shell poly (methyl methacrylate) latex colloids, Langmuir 19, 5963 (2003).

[61] S. M. Klein, V. N. Manoharan, D. J. Pine, and F. F. Lange, Preparation of monodisperse PMMA microspheres in nonpolar solvents by dispersion polymerization with a macromonomeric stabilizer, Colloid Polym. Sci. 282, 7 (2003).

[62] https://github.com/Dullens-Lab/BLoSSOM. 